is offered using distraction tests at 7 months and audiometric assessments at school entry and in alternate academic years until school leaving age. This would seem to give comprehensive coverage, but in practice variations arise: some tests are postponed due to illness or absenteeism and children screened at the beginning of one academic year may not be recalled until the end of the academic year when further testing is scheduled, an interval approaching three years. A period of this duration could be of long term consequence for a young child with unrecognised unilateral hearing loss.

Our investigation indicated difficulty in attributing a cause for sensorineural hearing loss in many of the cases identified. In 11 the defect was described as congenital despite available evidence of previously normal hearing and in 16 no conclusion was reached. Delay in ascertainment of hearing impairment, which may have been unsuspected by parents, and the extent of credence given to hearing testing in the community by hospital staff are both factors that might hamper diagnosis.

Temporal evidence suggests that mumps could be considered as a cause in a third of the cases of hearing loss studied. Subclinical or unrecognised mumps may have occurred in other instances. Seven cases contracted mumps between 1 April 1981 and 31 March 1982, in association with a nationally observed periodic rise in incidence. ${ }^{6}$ The possibility that particular strains of mumps virus might be more prone to cause auditory damage requires consideration.

The incidence of hearing loss due to mumps is unclear as few studies are available, particularly in relation to young children. This investigation concludes that mumps could be considered more widely as an explanation for some cases of unilateral hearing loss and that a formal prospective study with serology is indicated. Meanwhile, the changing epidemiology of mumps to a milder illness of younger children, the fact that permanent hearing impairment can result from subclinical or unrecognised infections, and the importance of unilateral hearing loss in this age group are factors that confirm the need for regular screening of hearing. In addition, routine testing of hearing should be considered after a history of mumps, particularly in districts where a comprehensive screening programme is not available.

\section{References}

1 Galbraith NS, Pusey JJ, Young SEJ, Crombie DL, Sparks JP. Mumps surveillance in England and Wales 1962-1981. Lancet 1984;i:91-4.

${ }^{2}$ Lindsay JR, Davey PR, Ward PH. Inner ear pathology in deafness due to mumps. Annals of Otolaryngology 1960;69:918.

3 Vuori M, Lahikainen EA, Pettonen T. Perceptive deafness in connection with mumps. Acta Otolaryngol (Stockh) 1962; 55:231-6.

${ }^{4}$ Fowler ER. Deafness in mumps. Pediatrics 1960;77:243-5.

5 Jones DT. Annual statistical report for the year ended 31/12/84. Mold, Clwyd, Wales: Clwyd Health Authority, 1985.

- Communicable Disease Surveillance Centre. Mumps surveillance 1969-83. Communicable Disease Report 1984;No 51.

Correspondence to Dr R Hall, Clwyd Health Authority, Preswylfa, Hendy Road, Mold, Clwyd CH7 1PZ, Wales.

Received 9 September 1986

\title{
Meningitis presenting as hypertension
}

\section{K WATERS AND J GILLIS}

\section{Intensive Care Unit, Royal Alexandra Hospital for Children, Sydney, Australia}

SUMMARY A 21 month old girl who presented with what seemed to be hypertensive encephalopathy is described. Although her encephalopathy resolved with antihypertensive treatment, subsequent investigations revealed haemophilus meningitis.

We report a case of a child with otherwise uncomplicated meningitis who presented with fever, systemic hypertension, and encephalopathy. The possible pathogenic mechanisms are discussed and the need to consider meningitis in the differential diagnosis of acute hypertension is emphasised.

\section{Case report}

A 21 month old girl was referred because of acute systemic hypertension associated with fever and drowsiness. There was no important history. A maternal aunt had had unspecified renal disease. The present admission had been preceded by three days of coryzal symptoms with no systemic illness. On the morning of admission she developed fever associated with deterioration in conscious state. She vomited a single dose of oral antibiotic but had not received antipyretics.

On examination at this time she was drowsy but rousable, with a temperature of $37.5^{\circ} \mathrm{C}$ (from 
previously measured $39^{\circ} \mathrm{C}$ ) and blood pressure $200 / 100 \mathrm{~mm} \mathrm{Hg}$. Peripheral pulses and perfusion were normal. There was no evidence of dehydration. Mild neck stiffness was noted. The fundi were normal with no evidence of papilloedema. There were no focal neurological signs, although there was generalised hypotonicity. There was no peripheral oedema and urinalysis revealed a trace of protein, moderate ketones, and two epithelial cells. There was a single cafe au lait spot. Abdominal examination yielded normal results. Laboratory investigations revealed no abnormalities and included a haemoglobin concentration of $11.8 \mathrm{~g} / \mathrm{dl}$, white blood cell count $10 \times 10^{9} / 1$, and normal creatinine and electrolyte concentrations. Chest $x$ ray yielded normal results and blood and urine cultures were taken. On the basis of these features a provisional diagnosis of hypertensive encephalopathy was entertained and the hypertension was treated with frusemide $1 \mathrm{mg} / \mathrm{kg}$ and diazoxide $5 \mathrm{mg} / \mathrm{kg}$ (in divided boluses over 60 minutes). Over the subsequent 30 minutes her blood pressure fell to $150 / 80 \mathrm{~mm} \mathrm{Hg}$, and this fall in blood pressure was associated with an improved conscious state. She was then transferred to our hospital.

On assessment after transfer her blood pressure was stable at $110 / 70 \mathrm{~mm} \mathrm{Hg}$. Blood pressure was equal in both upper and lower limbs. Her temperature remained raised at $38.8^{\circ} \mathrm{C}$. Her conscious state had improved to appropriate verbal responses, although she remained irritable. Fundi were normal but neck stiffness was noted and there was mild peripheral weakness. There was no evidence of dysautonomia. Repeat full blood count showed a haemoglobin concentration of $13.9 \mathrm{~g} / \mathrm{dl}$ and a white blood cell count of $20 \times 10^{9} / 1$ with toxic changes. In view of the neck stiffness and now toxic blood film a lumbar puncture was performed, which showed a turbid cerebrospinal fluid under normal pressure with 7600 white cells $/ \mathrm{ml}, 2400$ red cells $/ \mathrm{ml}$, and Gram negative bacilli. She was begun on treatment with ampicillin and chloramphenicol and slightly restricted intravenous fluids.

Throughout her hospital stay blood pressure remained within the normal range. Temperature and neurological examination became normal within 48 hours and remained so. Cerebrospinal fluid cultures grew Haemophilus influenzae type b. Serum creatinine, urea, and electrolyte concentrations were normal and renal ultrasound showed normal renal anatomy.

\section{Discussion}

This child is presented to illustrate an unusual presentation of acute meningitis. Although acute transient hypertension is known to occur in diseases associated with raised intracranial pressure, ${ }^{1}$ the association with acute meningitis is not documented. There was no clear evidence in our patient for raised intracranial pressure. Her fundi were normal, there were no suggestive neurological signs, and cerebrospinal fluid was tapped at normal pressure on lumbar puncture. At the time of presentation her encephalopathy was considered to be secondary to systemic hypertension, and this seemed to be supported when her conscious state improved after treatment of the hypertension. The dose of diuretic that she received, however, may have resolved a raised intracranial pressure that was not clinically evident.

Whatever the specific pathophysiology, the hypertension in our patient does seem to have been associated with the haemophilus meningitis. There was neither evidence for nor treatment of extracellular fluid volume depletion. There was no suggestion of Riley-Day syndrome. Our investigations cannot, however, totally exclude renal disease or primary catecholamine excess, although neither seem probable.

The pathophysiology of meningitis involves diverse mechanisms that could produce a picture of hypertension and encephalopathy.

(1) The involvement of blood pressure regulatory centres from the hypothalamus and medulla. ${ }^{2-4}$ For example, (a) ischaemia or hypoxia of medullary centres through blood flow alterations associated with inflammation or ischaemia produced by mechanical compression $;^{3-5}$ (b) increased blood volume through hypothalamic antidiuretic hormone mechanisms, although usually this would involve a longer time course $;^{5-6}$ (c) sympathetic overactivity through stimulation of brain stem or spinal centres in which there may be other evidence of autonomic malfunction, such as poor peripheral perfusion, tachycardia, or poor urine output. ${ }^{56}$

(2) Raised intracranial pressure, although this would usually be associated with vagal activity. ${ }^{4}$

(3) Some children may have an underlying idiopathic tendency to hypertension in stressful situations. ${ }^{1}$

It is not possible to delineate the exact mechanism of our patient's hypertension. Although mild raised intracranial pressure was possibly the predominant cause, this was certainly not detectable clinically apart from encephalopathy originally attributed to systemic hypertension. The assumption that systemic hypertension in cases of meningitis is secondary to raised intracranial pressure is therefore questionable. It is also evident that meningitis should always be considered in the differential diagnosis of acute systemic hypertension, especially if this is associated 
with fever. This case also illustrates the potential benefit in treating hypertension even in acute neurological disorders, although this should always be performed cautiously. ${ }^{6}$

\section{References}

${ }^{1}$ Loggie JM. Systemic hypertension in children and adolescents. Pediatr Clin North Am 1971;18:1273-310.

2 Wilkins RH, Rengachary SS. Neurosurgery. New York: McGraw-Hill, 1985.

${ }^{3}$ Nagao S, Sunami N, Tsutsui $T$, et al. Acute intracranial hypertension and brain-stem blood flow. J Neurosurg 1984;60:566-71.
4 Evans A. Cerebral ischaemia as a factor in the vasomotor response to increased intracranial pressure. Tex Med 1967;63:84-90.

5 Scheld WM. Pathophysiological correlates in bacterial meningitis. Journal of Infection 1981;3 (Supp 1):5-9.

${ }^{6}$ Eden OB, Sills JA, Brown JK. Hypertension in acute neurological diseases in childhood. Dev Med Child Neurol 1977;19:437-45.

Correspondence to Dr J Gillis, Intensive Care Unit, Royal Alexandra Hospital for Children, Pyrmont Bridge Road, Camperdown, New South Wales, 2050, Australia.

Received 1 August 1986

\title{
Phosphatidylglycerol in tracheal aspirates for diagnosis of hyaline membrane disease
}

\author{
J FRANCOUAL, J F MAGNY, J C ROPERT, M DEHAN, AND R LELUC
}

Laboratoire de Biochimie and Service de Réanimation Néonatale, Hôpital Antoine Béclère, Clamart, France

SUMMARY Lecithin:sphingomyelin ratio and phosphatidylglycerol were determined by a rapid, simple method in tracheal aspirates obtained from 132 newborn infants with respiratory diseases, sixty five of whom developed hyaline membrane disease. Phosphatidylglycerol determination was more sensitive $(97 \%)$ than lecithin:sphingomyelin ratio, but their specificities were similar $(76 \%)$.

A deficiency in the pulmonary surfactant is believed to cause hyaline membrane disease in newborn infants. Pulmonary maturity has been assessed by the analysis of the phospholipids in the hypopharyngeal ${ }^{1}$ and tracheal ${ }^{2}$ aspirates of newborn infants. The most widely used test is the lecithin: sphingomyelin ratio described by Gluck et al, ${ }^{3}$ but this method is time consuming and affected by contamination of the specimen with blood or meconium.

As a consequence, several investigators have suggested that other phospholipids from surfactant and particularly phosphatidylglycerol (PG) should be studied. ${ }^{4} \mathrm{PG}$ is synthesised almost exclusively by lung alveolar cells so that there is no interference in assays by blood or meconium. Both one and two dimensional thin layer chromatography are commonly used to analyse PG. These methods are time consuming, however, and require extensive laboratory technician training.

In 1983. Garite et al described a new, rapid, simple, and reliable method to determine $P G$ in amniotic fluid by a semiquantitative immunological slide agglutination test. ${ }^{5} \mathrm{~A}$ complete assay can be performed in about 30 minutes.

We have applied and compared this test to the lecithin:sphingomyelin in the tracheal aspirate.

\section{Patients and methods}

Tracheal aspirates were obtained from 132 infants who were admitted to the intensive care unit for respiratory disease. Of these, 65 were diagnosed as having hyaline membrane disease after satisfying clinical and $x$ ray criteria. The other 67 infants had other respiratory diseases-namely, transient tachypnoea of the newborn (30 cases), congenital pneumonia (six), amniotic fluid aspiration syndrome (11), and other respiratory diseases (20). The gestational ages of the infants ranged from 26 to 42 weeks (mean 33 weeks) and their birth weights from 645 to $4700 \mathrm{~g}$ (mean $2160 \mathrm{~g}$ ). Samples were obtained during the first 24 hours after birth.

The lecithin:sphingomyelin ratio was determined using the method of Gluck $e t \mathrm{al}^{3}$ and PG testing by Amniostat-FLM ${ }^{5}$ were performed on all samples. A lecithin:sphingomyelin ratio $\geqslant 2.0$ and a $P G \geqslant++$ or $2 \mu \mathrm{g} / \mathrm{ml}$ were interpreted as being 'positive' and as indicating fetal lung maturity and a lecithin:sphingomyelin ratio $<2$ and $\mathrm{PG}<++$ were interpreted as being 'negative'. ${ }^{2-5}$

\section{Results}

The Table shows the results of the lecithin:sphin- 